INPLASY

PROTOCOL

To cite: Zhang et al. Can oral dietary nutrition prevent cancer chemotherapy-induced cardiotoxicity? An evidence mapping of published in vivo records. Inplasy protocol 202230015. doi:

10.37766/inplasy2022.3.0015

Received: 03 March 2022

Published: 03 March 2022

Corresponding author: Quan Wang

wangquan2013@126.com

Author Affiliation:

Ambulatory Surgery Center, Xijing Hospital, Air Force Military Medical University, Xi'an 710032, China.

Support: None.

Review Stage at time of this submission: Ongoing.

Conflicts of interest:

None declared.

\section{Can oral dietary nutrition prevent cancer chemotherapy-induced cardiotoxicity? An evidence mapping of published in vivo records}

Zhang, XY1; Yang, KL2; Liu, XN3; Li, Y4; Zhao, Y5; Jiang, KW6; Wang, $Q^{7}$.

Review question / Objective: We aimed to detect the effectivenss of oral dietary nutrition against cancer chemotherapy-induced cardiotoxicity based on in vivo studies.

Condition being studied: Cancer chemotherapy-induced cardiotoxicity face the risk of severe poor prognoses for cancer survivors. It is significantly important to seek a more cost effective cardiac protection strategy. However, the current evidence on the effectiveness of nutritional intervention against cancer chemotherapy-related cardiotoxicity was limited on account of the lack of enough human-level studies. In addition, the guidelines failed to report the aspect of nutrition against cancer chemotherapyinduced cardiotoxicity in detail. Some published reviews about this topic did not involve all types of nutrition which was relevant to human diet.

INPLASY registration number: This protocol was registered with the International Platform of Registered Systematic Review and Meta-Analysis Protocols (INPLASY) on 03 March 2022 and was last updated on 03 March 2022 (registration number INPLASY202230015).

\section{INTRODUCTION}

Review question / Objective: We aimed to detect the effectivenss of oral dietary nutrition against cancer chemotherapyinduced cardiotoxicity based on in vivo studies.
Condition being studied: Cancer chemotherapy-induced cardiotoxicity face the risk of severe poor prognoses for cancer survivors. It is significantly important to seek a more cost effective cardiac protection strategy. However, the current evidence on the effectiveness of nutritional intervention against cancer 
chemotherapy-related cardiotoxicity was limited on account of the lack of enough human-level studies. In addition, the guidelines failed to report the aspect of nutrition against cancer chemotherapyinduced cardiotoxicity in detail. Some published reviews about this topic did not involve all types of nutrition which was relevant to human diet.

\section{METHODS}

Participant or population: Healthy or tumorbearing animal models undergoing chemotherapy treatment, regardless of the species, gender, age and body weight of animals and the drug and dose of chemotherapy.

Intervention: Oral dietary nutrition including natural food or food extract, but excluding drugs and non-foodextract.

Comparator: Standard chow diet without nutrition above.

Study designs to be included: In vivo studies.

Eligibility criteria: (a) participants: healthy or tumor-bearing animal models undergoing chemotherapy treatment, regardless of the species, gender, age and body weight of animals and the drug and dose of chemotherapy; (b) intervention: oral dietary nutrition including natural food or food extract, but excluding drugs and nonfood extract; (c) comparison: standard chow diet without nutrition above; (d) outcomes: at least one indicator of chemotherapy-induced cardiotoxicity, which included ultrasonic cardiogram (UCG, including left ventricular ejection fraction (LVEF), left ventricular fractional shortening (LVFS), left ventricular endsystolic diameter (LVESD), left ventricular end-diastolic diameter (LVEED)), serum cardiac markers (creatine kinase (CK), creatine kinase-MB (CK-MB), lactate dehydrogenase (LDH), aspartate transaminase (AST), brain natriuretic peptide (BNP), N-terminal pro-brain natriuretic peptide (NT-proBNP), cardiac troponin (cTn)), oxidative stress markers (malondialdehyde (MDA), superoxide dismutase (SOD), glutathione (GSH), glutathione peroxidase (GPX), catalase (CAT)), and histopathological examination.

Information sources: Pubmed, Embase, the Cochrane Library and grey literature.

Main outcome(s): ultrasonic cardiogram (UCG, including left ventricular ejection fraction (LVEF), left ventricular fractional shortening (LVFS), left ventricular endsystolic diameter (LVESD), left ventricular end-diastolic diameter (LVEED)), serum cardiac markers (creatine kinase (CK), creatine kinase-MB (CK-MB), lactate dehydrogenase (LDH), aspartate transaminase (AST), brain natriuretic peptide (BNP), N-terminal pro-brain natriuretic peptide (NT-proBNP), cardiac troponin (cTn)), oxidative stress markers (malondialdehyde (MDA), superoxide dismutase (SOD), glutathione (GSH), glutathione peroxidase (GPX), catalase (CAT)), and histopathological examination.

Quality assessment / Risk of bias analysis: Risk of bias of included studies was assessed by two reviewers independently using the Systematic Review Center for Laboratory Animal Experimentation's (SYRCLE) risk of bias tool. This tool was developed based on Cochrane Risk of Bias (RoB) tool for animal experiments and consisted of 10 items involving selection bias, performance bias, detection bias, attrition bias, reporting bias and other biases.

Strategy of data synthesis: Indicators which were reported most in three types of outcome measures (UCG, serum cardiac markers and oxidative stress markers) were selected and shown by using histograms of Chemo/CON (the data ratio of chemotherapy group to control group) and Chemo+Nutr/CON (the data ratio of chemotherapy with oral dietary nutrition group to control group).

Subgroup analysis: None.

Sensitivity analysis: None. 
Country(ies) involved: China and Belgium.

Keywords: chemotherapy; cardiotoxicity; heart diseases; oral nutrition; diet therapy; systematic review.

Contributions of each author:

Author 1 - Xinyu Zhang - The author searched literatures and drafted the manuscript.

Email: 10159558@boe.com.cn

Author 2 - Kelu Yang - The author searched literatures and drafted the manuscript.

Email: kelu.yang@kuleuven.be

Author 3 - Xiaonan Liu - The author provided statistical expertise.

Email: liuxnxjh@163.com

Author 4 - Yang Li - The author provided statistical expertise.

Email: liyang2016@bjmu.edu.cn

Author 5 - Yang Zhao - The author read, provided feedback and approved the final manuscript.

Email: m13893604689@163.com

Author 6 - Kewei Jiang - The author read, provided feedback and approved the final manuscript.

Email: jiangkewei@pkuph.edu.cn

Author 7 - Quan Wang - The author proposed the idea, read, provided feedback and approved the final manuscript.

Email: wangquan2013@126.com 